\title{
Effect of Repetitive Transcranial Magnetic Stimulation on the Right Superior Temporal Gyrus for Severe Aphasia Caused by Damage to the Left Inferior Frontal Gyrus
}

\author{
Mimpei Kawamura Nobuhiro Takahashi Yasutaka Kobayashi \\ Department of Rehabilitation, Speech-Language-Hearing Therapy, Fukui Health Science \\ University, Fukui, Japan
}

\section{Keywords}

Aphasia $\cdot$ rTMS · Superior temporal gyrus

\begin{abstract}
Several reports on repetitive transcranial magnetic stimulation (rTMS) for the treatment of aphasia caused by damage to the left inferior frontal gyrus state that low-frequency rTMS therapy for the right inferior frontal gyrus, which is contralateral to the focus area, is effective for improving verbal expression. However, most of these reports have studied the effects of rTMS therapy for comparatively mild aphasia. This study attempted to perform low-frequency rTMS on the right posterior superior temporal gyrus (BA22), which is the center for language reception for aphasia patients with a drastic decline in verbal expression due to damage to the left inferior frontal gyrus and a considerable decline in language perception. The participants performed a language task that was displayed on a computer monitor during rTMS. In addition, intensive speech-language and hearing therapy was performed by the therapist after rTMS. This study reports that a resultant improvement in language perception was observed
\end{abstract}




\section{Case Reports in Neurology}

Case Rep Neurol 2019;11:189-198

DOI: $10.1159 / 000500669$

2019 The Author(s). Published by S. Karger AG, Basel www.karger.com/crn

Kawamura et al.: Repetitive Transcranial Magnetic Stimulation for Severe Aphasia

in the activated brain regions based on neuropsychological tests and functional magnetic resonance imaging. This study is considered to be significant as it highlights a new method of rTMS treatment for severe aphasia.

(C) 2019 The Author(s)

Published by S. Karger AG, Basel

\section{Introduction}

Repetitive transcranial magnetic stimulation (rTMS) can alter the excitability of the cerebral cortex by altering the stimulus intensity, stimulus frequency, and the number of applications. Low-frequency rTMS with a maximum of $1 \mathrm{~Hz}$ effectively suppresses the stimulation site, and high-frequency rTMS with a minimum of $5 \mathrm{~Hz}$ can effectively excite it. In this manner, rTMS can alter the excitability of the cerebral cortex. There are reports on the practical applications of rTMS for treating various central nervous system diseases, by stimulating the cerebrum, which has a complex network that responds to excitability and suppression, using combinations of different methods.

Heiss and Thiel [1] reported that the right inferior frontal gyrus (RIFG) becomes overactive in aphasia due to damage to the left inferior frontal gyrus (LIFG). They extrapolated that this overactivation of the RIFG is a maladaptive response caused by interhemispheric suppression of the LIFG, which hinders its functional recovery. Naeser et al. [2] performed lowfrequency rTMS on the right frontal lobe for chronic stroke patients with aphasia due to damage to the left frontal lobe. Results indicated that low-frequency rTMS improved aphasia in such patients, confirmed by neuropsychological tests. This improvement is thought to result from the liberation of the left hemisphere from interhemispheric suppression, leading to its appropriate activation brought about by suppressing activation of the right frontal lobe. Recent reports have confirmed a significant recovery in speech mechanisms when rTMS and intensive speech-language and hearing therapy (iST) were used for the RIFG of chronic stroke patients, which is contralateral to the area of focus, in comparison with chronic stroke patients, in whom sham rTMS and iST were used [3]. Furthermore, coadministration of low-frequency rTMS to the RIFG (BA45) and language training for patients exhibiting conduction aphasia caused by damage to the left posterior region has led to reports that confirm activation of not only the left frontal language region, but also the left posterior region (surrounding BA22) [4]. Although these methods recognize the effectiveness of rTMS to the RIFG, it is limited to treating comparatively mild aphasia with nonfluent utterances that limit language expression, rather than language reception. There are fewer reports on its curative effects in aphasia patients with a considerable decline in language reception. Language processing within the brain was conventionally thought to include language reception (input) functions, such as listening and reading and language expression (output) functions, such as speaking and writing. The bidirectional input and output network enables smooth language communication. Anatomically, the arcuate fasciculus is a bundle of nerve fibers that are essential for this network. Several patients with expressive aphasia, with minimum-to-moderate damage to the frontal region, experience difficulty in both output and input functions. Considering the hierarchical nature of language, it is preferable that rehabilitation programs prioritize the improvement of input abilities in these patients. For the recovery of aphasia after stroke, it is crucial to mobilize the functions of the left superior temporal gyrus (BA22) and its 


\section{Case Reports in Neurology}

Case Rep Neurol 2019;11:189-198

DOI: $10.1159 / 000500669$

2019 The Author(s). Published by S. Karger AG, Base www.karger.com/crn

Kawamura et al.: Repetitive Transcranial Magnetic Stimulation for Severe Aphasia

surrounding regions during the recovery of input abilities. Therefore, we assume that the application of inhibitory stimulation to the contralateral side, that is the superior temporal gyrus (BA22), similar to the inhibitory application of rTMS to BA45, contralateral to the focus area, for aphasia patients with input and output speech restrictions may potentially improve their input abilities. In this study, we observed improved language reception due to inhibitory rTMS on BA22, contralateral to the focus area, in chronic stroke patients with moderate-to-severe aphasia.

\section{Case Presentation}

A 60-year-old male had right hemiplegia caused by left internal carotid artery occlusion secondary to cardiogenic embolism (time after onset: 42 months). The Brunnstrom stages of recovery were as follows: upper limb, II; finger, II; and lower limb, II. He exhibited language processing disorders with severe expressive aphasia and moderate receptive aphasia (Table 1 , SLTA Pre). The subject is right-handed but uses his left hand. T1 axial image confirmed a large area of damage around the LIFG (Fig. 1).

Pre- and Post-Tests

Neuropsychological Examination

We performed the Standard Language Test of Aphasia (SLTA) [5]. We also performed a deep test for language reception (listening) by testing noun recognition based on the semantic category, which is a subset of the Test of Lexical Processing in Aphasia (TLPA). We calculated the correct answer rate and $z$-score [6]. The pre-test was performed 2-3 days before the patient received treatment, and the post-test 1-2 days after completion of treatment.

\section{Brain Function Measurement}

To measure language processing activities in the brain, before and after treatment, we performed fMRI using the 1.5T Optima MR360 (GE Healthcare, USA). The imaging parameters were: repetition time 3,000 $\mathrm{ms}$ ( $1 \mathrm{scan}$ ), echo time $=40 \mathrm{~ms}$, axial slice $=32$ slices, flip angle $90^{\circ}$, field of view $256 \times 256 \mathrm{~mm}$, matrix $=64 \times 64$, slice thickness $=4 \mathrm{~mm}$, slice gap $=0 \mathrm{~mm}$, and 135 volumes/session. We excluded the first 5 volumes from the data analysis in an attempt to stabilize the magnetic susceptibility.

We implemented a six-block block design (two conditions, three blocks) with $30 \mathrm{~s}$ of tasks and $30 \mathrm{~s}$ of rest. We tested the following two conditions: (i) listening to meaningful words was the task condition, and (ii) listening to meaningless sounds (clicking sounds) was the control condition. Using headphones (Serene Sound; Resonance Technology), we checked the sound volume before proceeding with the measurements. Task conditions included an interval of $2 \mathrm{~s}$ for 15 words. We used high-frequency words, which were chosen from a total of 27,596 words between 5.001 and 7.000 for word familiarity in the NTT Database Series Nihongo-no Goi-Tokusei [7]. The spoken words were recorded in standard Japanese by a professional (female) narrator.

We conducted an analysis using SPM8 (Statistical Parametric Mapping). As preprocessing, we performed realignment, estimation of normalization, smoothing (full width half 
maximum: $\mathrm{x}=6 \mathrm{~mm}, \mathrm{y}=6 \mathrm{~mm}, \mathrm{z}=6 \mathrm{~mm}$ ), and temporal filtering (high-pass filter, $0.01 \mathrm{~Hz}$ ). We calculated the activation site by subtracting the control conditions from the task conditions. The level of significance was 1\% (uncorrected), and the coordinates where the cluster size had a level of significance of approximately $5 \%$ were related areas. Second, the coordinates of related areas were converted from Montreal Neurological Institute (MNI) coordinates to Talairach coordinates [8], and the corresponding brain regions were isolated.

\section{Repetitive Transcranial Magnetic Stimulation}

We used figure-8 coils (Magstim, UK) to perform low-frequency rTMS (1 Hz), with 1,200 applications, twice a day for 10 days. The stimulation intensity was $90 \%$ of the threshold of the motor-evoked potentials during rest, which was derived through the abductor pollicis brevis muscle of the left hand. The stimulation threshold was BA22, which was identified by the navigation system. Moreover, we provided language stimulation from a computer monitor to stimulate language reception during rTMS. We encouraged the subject to watch the computer monitor, where a series of words were displayed, with 1 word displayed for $20 \mathrm{~s}$ (picture-naming cards A [4 s]; Chinese characters that indicate A [4 s]; kana syllabaries that indicate A [4 s]; picture-naming cards [4 s]; and blank cards [4 s]). We auto-played 60 words for 20 min using Microsoft Office PowerPoint.

\section{Administration of iST}

We conducted iST for $60 \mathrm{~min}$, twice a day for 10 days, based on the results of the neuropsychological examinations. Speech-language-hearing therapists conducted iST immediately after rTMS. We conducted iST for the following: (1) auditory comprehension issues; (2) semantic therapy (nouns, verbs); and (3) language reception (listening, reading) such as semantic word problems. The content and difficulty level were changed, whenever appropriate.

\section{Results}

The SLTA pretest confirmed a severe decline in language expression for speaking and writing. It also indicated a moderate decline in language reception for listening and reading. The post-test did not demonstrate any remarkable changes in comparison to the pretest (Table 1). However, the TLPA, which is a deep test for listening, confirmed a clear improvement for all categories, excluding body regions and color. The correct answer rate for the pretest was $53.5 \%$ with a $z$-score of -1.66 , whereas the post-test had a correct answer rate of $69.5 \%$, with a $z$-score of -0.73 , which confirms a remarkable improvement in listening grades (Table 2).

The fMRI results are shown in Table 3. The pretest confirmed significant stimulation in the superior, middle, and inferior temporal gyrus (STG, MTG, ITG) for the left hemisphere $(p<0.001$, uncorrected). Significant stimulation was observed in the MTG for the right hemisphere ( $p<0.001$, uncorrected). The post-test confirmed greater stimulation in the left and right temporal gyrus. However, significant stimulation was confirmed in a wider brain region for the ITG in the post-test than in the pretest ( $p<0.001$, uncorrected). Furthermore, significant stimulation was also confirmed in the posterior cingulate gyrus (BA31) and thalamus for the left hemisphere $(p<0.001$, uncorrected). 


\section{Case Reports in Neurology}

\section{Discussion}

Language function in the brain is hierarchical. The frontal region of the brain controls the output function, while the posterior region controls the input function. This bidirectional network enables smooth language communication. Therefore, many patients with motor aphasia caused by damage to the frontal region have difficulty in output and input functions. Considering this structure of language, training programs should prioritize improving input function for rehabilitation.

We conducted rTMS and iST by prioritizing the sim to enhance the functions of the posterior region, rather than focusing on improving the functions originally possessed by the frontal region. Hence, we obtained a remarkable improvement in input functions, as shown by the improved TLPA score. Neuroscientific evidence was provided by the fMRI as well, which showed increased intensity and expansion of range in the periphery of Wernicke's area, which is the center of input function, as well in activities in MTG and ITG, which store longterm language information.

The post-test recognized stimulation in the posterior cingulate gyrus (BA31) and thalamus. According to Pandya and Yeterian [9], BA31 has fibers that intermingle with the multisensory association cortex, such as the MTG, with the thalamus. Furthermore, fiber intermingling is seen with the limbic system, which is closely related to long-term memory $[10,11]$. Grasby et al. [12] observed increased blood flow in the posterior cingulate gyrus including BA31 when subjects were assigned the task of memorizing and recalling words that have been read out loud [12]. Thus, BA31 controls long-term memory and contributes to understanding input information. Moreover, damage to the left thalamus causes semantic memory disorders and hinders access to stored vocabulary $[13,14]$. The activation of the left thalamus in this study is considered to be scientific evidence that it controls the access to stored vocabulary.

rTMS with language stimulation is a new method, which has not been reported previously. Meanwhile, external language stimulation was provided so that the language center could be stimulated efficiently, which may have produced stable and more efficient plastic changes to the nerves. Future studies are needed, as this is currently conjecture.

Finally, this report performed rTMS to BA22 for patients with severe aphasia due to leftanterior-region injury. In addition, speech therapy was performed during and after rTMS. A similar attempt has never been reported, and this could be a significant future treatment for aphasia.

\section{Acknowledgements}

We thank the aphasic patient and his family for their help in this study. In addition, we would like to thank the therapist in charge of the aphasia patient. 


\section{Statement of Ethics}

Informed consent was obtained from the patient and the family for the publication of this report. This study was conducted ethically in accordance with the Helsinki Declaration. In addition, the research protocol has been approved by the ethics committee to which it belongs.

\section{Disclosure Statement}

The authors declare that they have no conflicts of interest to disclose.

\section{Author Contributions}

All persons who meet authorship criteria are listed as authors, and all authors certify that they have participated sufficiently in the work to take public responsibility for the content, including participation in the concept, design, analysis, writing, or revision of the manuscript. Furthermore, each author certifies that this material or similar material has not been and will not be submitted to or published in any other publication before its appearance in Case Reports in Neurology.

Mimpei Kawamura (corresponding author) contributed to the following four conditions.

1 Substantial contributions to the conception or design of the work; or the acquisition, analysis, or interpretation of data for the work.

2 Drafting the work or revising it critically for important intellectual content.

3 Final approval of the version to be published.

4 Agreement to be accountable for all aspects of the work in ensuring that questions related to the accuracy or integrity of any part of the work are appropriately investigated and resolved.

Nobuhiro Takahashi (co-author) contributed to the following four conditions.

1 Substantial contributions to the conception or design of the work; or the acquisition, analysis, or interpretation of data for the work.

2 Drafting the work or revising it critically for important intellectual content.

3 Final approval of the version to be published.

4 Agreement to be accountable for all aspects of the work in ensuring that questions related to the accuracy or integrity of any part of the work are appropriately investigated and resolved.

Yasutaka Kobayashi (co-author) contributed to the following four conditions.

1 Substantial contributions to the conception or design of the work; or the acquisition, analysis, or interpretation of data for the work.

2 Drafting the work or revising it critically for important intellectual content.

3 Final approval of the version to be published.

4 Agreement to be accountable for all aspects of the work in ensuring that questions related to the accuracy or integrity of any part of the work are appropriately investigated and resolved. 


\section{References}

1 Heiss WD, Thiel A. A proposed regional hierarchy in recovery of post-stroke aphasia. Brain Lang. 2006 Mar;98(1):118-23.

2 Naeser MA, Martin PI, Nicholas M, Baker EH, Seekins H, Kobayashi M, et al. Improved picture naming in chronic aphasia after TMS to part of right Broca's area: an open-protocol study. Brain Lang. 2005 Apr;93(1):95-105.

3 Haghighi M, Mazdeh M, Ranjbar N, Seifrabie MA. Further evidence of the positive influence of repetitive transcranial magnetic stimulation on speech and language in patients with aphasia after stroke: results from a double-blind intervention with sham condition. Neuropsychobiology. 2017;75(4):185-92.

4 Zhang H, Chen Y, Hu R, Yang L, Wang M, Zhang J, et al. rTMS treatments combined with speech training for a conduction aphasia patient: A case report with MRI study. Medicine (Baltimore). 2017 Aug;96(32):e7399.

5 Japan Society for Higher Brain Dysfunction. Standard language test of aphasia. Tokyo: Shinko Igaku Syuppansya; 2006.

6 Fujita I, Monoi H, Okudaira N, Ueda M, Ono K, Shimogaki Y, et al. A test of lexical processing in aphasia. Tokyo: Esco Earl; 2000.

7 Amano N, Kasahara K, Kondo T. NTT Database Series Nihongo-no Goi-Tokusei (Lexical properties of Japanese). Tokyo: Sanseido; 1999.

8 Talairach J, Tournoux P. Co-Planar Stereotactic Atlas of the Human Brain. Stuttgart, New York: Thieme; 1988.

9 Pandya DN, Yeterian EH. Architecture and connections of cortical association areas. In: Peters A, Jones EG, editors. Association and Auditory Cortices. Cerebral Cortex, Vol. 4. Boston: Springer; 1985. p. 3-61.

10 Pandya DN, Van Hoesen GW, Mesulam MM. Efferent connections of the cingulate gyrus in the rhesus monkey. Exp Brain Res. 1981;42(3-4):319-30.

11 Mufson EJ, Pandya DN. Some observations on the course and composition of the cingulum bundle in the rhesus monkey. J Comp Neurol. 1984 May;225(1):31-43.

12 Grasby PM, Frith CD, Friston KJ, Bench C, Frackowiak RS, Dolan RJ. Functional mapping of brain areas implicated in auditory—verbal memory function. Brain. 1993 Feb;116(Pt 1):1-20.

13 Mori E, Ishii K, Hashimoto M, Imamura T, Hirono N, Kitagaki H. Role of functional brain imaging in the evaluation of vascular dementia. Alzheimer Dis Assoc Disord. 1999 Oct-Dec;13 Suppl 3:S91-101.

14 Mori E, Yamadori A, Mitani Y. Left thalamic infarction and disturbance of verbal memory: a clinicoanatomical study with a new method of computed tomographic stereotaxic lesion localization. Ann Neurol. 1986 Dec;20(6):671-6.

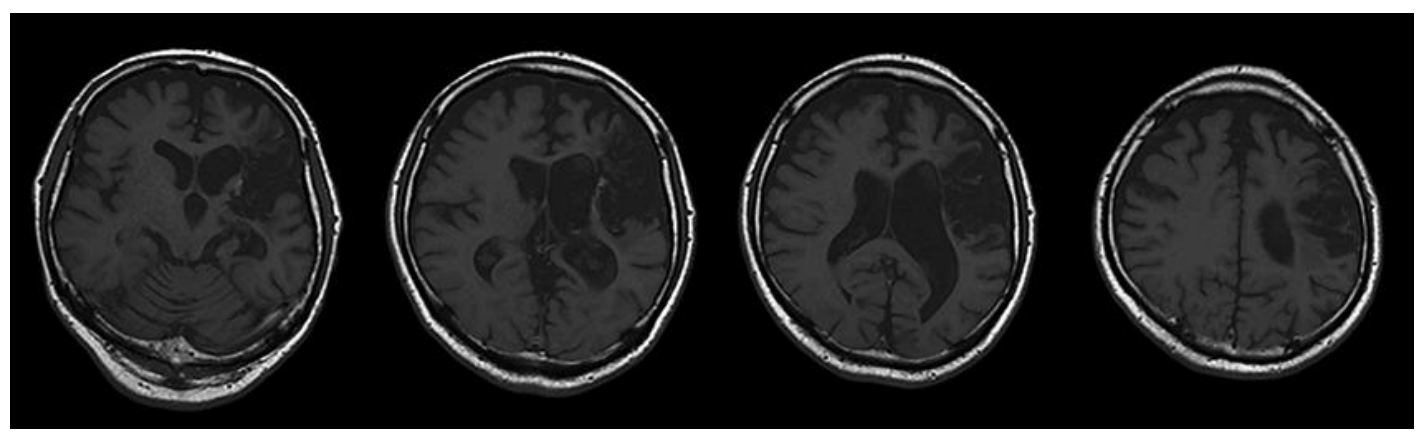

Fig. 1. T1 axial image (time after onset: 42 months). 
Table 1. Neuropsychological examination: SLTA

\begin{tabular}{|c|c|c|c|c|}
\hline & & & Corr & swers, \% \\
\hline & & & pre & post \\
\hline I & 1 & Auditory word recognition & 100 & 80 \\
\hline & 2 & Sentence comprehension & 60 & 60 \\
\hline & 3 & Follow verbal commands & 0 & 0 \\
\hline & 4 & Kana letter discrimination & 10 & 30 \\
\hline II & 5 & Speaking object naming & 0 & 0 \\
\hline & 6 & Word repetition & 30 & 20 \\
\hline & 7 & Describe behaviors & 0 & 0 \\
\hline & 8 & Explain picture story & 0 & 20 \\
\hline & 9 & Sentence repetition & 0 & 0 \\
\hline & 10 & Word fluency & 0 & 0 \\
\hline & 11 & Read aloud kanji words & 0 & 20 \\
\hline & 12 & Read aloud kana letters & 0 & 0 \\
\hline & 13 & Read aloud kana words & 20 & 0 \\
\hline & 14 & Read aloud short sentence & 0 & 0 \\
\hline III & 15 & Kanji word - picture matching & 80 & 100 \\
\hline & 16 & Kana word - picture matching & 70 & 80 \\
\hline & 17 & Sentence - picture matching & 70 & 50 \\
\hline & 18 & Follow written commands & 0 & 0 \\
\hline IV & 19 & Write kanji words & 0 & 20 \\
\hline & 20 & Write kana words & 0 & 0 \\
\hline & 21 & Narrative writing & 0 & 0 \\
\hline & 22 & Dictate kana letters & 20 & 0 \\
\hline & 23 & Dictate kanji words & 0 & 20 \\
\hline & 24 & Dictate kana words & 0 & 0 \\
\hline & 25 & Dictate short sentence & 0 & 0 \\
\hline
\end{tabular}

I, listening; II, speaking; III, reading; IV, writing. 
Table 2. Noun recognition based on semantic category: TLPA

\begin{tabular}{|c|c|c|c|c|c|c|c|}
\hline & \multirow{2}{*}{$\begin{array}{l}\text { All words, } \\
n\end{array}$} & \multicolumn{3}{|l|}{ Pre } & \multicolumn{3}{|l|}{ Post } \\
\hline & & $\begin{array}{l}\text { correct } \\
\text { answer, } n\end{array}$ & $\begin{array}{l}\text { correct } \\
\text { answer, \% }\end{array}$ & $z$-score & $\begin{array}{l}\text { correct } \\
\text { answer, } n\end{array}$ & $\begin{array}{l}\text { correct } \\
\text { answer, \% }\end{array}$ & $z$-score \\
\hline Indoor objects & 20 & 11 & 55.0 & -0.78 & 13 & 65.0 & -0.30 \\
\hline Structures & 20 & 16 & 80.0 & -0.12 & 17 & 85.0 & 0.14 \\
\hline Vehicles & 20 & 11 & 55.0 & -2.35 & 17 & 85.0 & -0.25 \\
\hline Tools & 20 & 11 & 55.0 & -1.69 & 18 & 90.0 & 0.18 \\
\hline Processed foods & 20 & 14 & 70.0 & -1.08 & 16 & 80.0 & -0.46 \\
\hline Vegetables and fruits & 20 & 8 & 40.0 & -2.39 & 12 & 60.0 & -1.32 \\
\hline Plants & 20 & 10 & 50.0 & -1.36 & 16 & 80.0 & 0.00 \\
\hline Animals & 20 & 11 & 55.0 & -2.07 & 16 & 80.0 & -0.56 \\
\hline Body parts & 20 & 6 & 30.0 & -1.87 & 5 & 25.0 & -2.08 \\
\hline Colors & 20 & 9 & 45.0 & -1.35 & 9 & 45.0 & -1.35 \\
\hline Total amount & 200 & 107 & 53.5 & -1.66 & 139 & 69.5 & -0.73 \\
\hline
\end{tabular}

z- scores were calculated based on the test data of 68 patients with aphasia, as indicated in the TLPA manual. 
Table 3. Activated brain areas on fMRI

\begin{tabular}{|c|c|c|c|c|c|c|c|}
\hline \multirow[t]{2}{*}{ Brain area } & & \multicolumn{3}{|c|}{ Talairach coordinate, $\mathrm{mm}$} & \multirow[t]{2}{*}{$z$-score } & \multirow[t]{2}{*}{$t$-score } & \multirow{2}{*}{$\begin{array}{l}\text { Cluster } \\
\text { size }\end{array}$} \\
\hline & & $\mathrm{x}$ & $\mathrm{y}$ & $\mathrm{z}$ & & & \\
\hline \multicolumn{8}{|l|}{ Pre } \\
\hline Left hemisphere & $\mathrm{BA}$ & & & & & & \\
\hline STG & 22 & -62 & -36 & 2 & 4.12 & 4.28 & 19 \\
\hline \multirow[t]{2}{*}{ MTG } & 21 & -58 & -22 & -14 & 4.50 & 4.70 & 95 \\
\hline & & -62 & -27 & -2 & 3.73 & 3.85 & \\
\hline ITG & 20 & -58 & -32 & -23 & 4.20 & 4.37 & 48 \\
\hline \multicolumn{8}{|l|}{ Right hemisphere } \\
\hline \multirow[t]{3}{*}{ MTG } & 21 & 60 & -18 & -11 & 4.35 & 4.53 & 51 \\
\hline & & 53 & -19 & -16 & 3.80 & 3.93 & \\
\hline & & 64 & -25 & -1 & 4.17 & 4.33 & 8 \\
\hline \multicolumn{8}{|l|}{ Post } \\
\hline \multicolumn{8}{|l|}{ Left hemisphere } \\
\hline STG & 22 & -62 & -23 & 0 & 3.51 & 3.61 & 13 \\
\hline \multirow[t]{2}{*}{ MTG } & 21 & -56 & -20 & -8 & 4.96 & 5.24 & 93 \\
\hline & & -60 & -15 & -12 & 3.74 & 3.86 & \\
\hline \multirow[t]{2}{*}{ ITG } & 20 & -58 & -28 & -21 & 3.84 & 3.97 & 39 \\
\hline & & -51 & -15 & -23 & 3.80 & 3.92 & 16 \\
\hline Posterior cingulate gyrus & 31 & -6 & -26 & 38 & 4.69 & 4.93 & 23 \\
\hline Thalamus & & -1 & -17 & 12 & 3.90 & 4.03 & 7 \\
\hline \multicolumn{8}{|l|}{ Right hemisphere } \\
\hline MTG & 21 & 55 & -20 & -13 & 4.81 & 5.06 & 56 \\
\hline
\end{tabular}

